\title{
La croyance de l'enfant selon Rousseau. Émile à la lumière des philosophie et psychologie de la religion
}

\section{Daniel Frey}

\section{Abstract}

The interest of the pages which Rousseau devotes in Emile to childhood belief has often been eclipsed by the famous Confession of faith of a Savoyard Vicar, which these pages are supposed to introduce. By leaving behind the narrow perspective of Rousseau Studies and adopting a double perspective of the psychology of religion and philosophy of religion, this article hopes to explain and discuss Rousseau's thesis that the child, incapable of conceiving the idea of God, does not believe so much in God as in the people who present the idea of God.

\section{Résumé}

L'intérêt des pages que Rousseau consacre à la croyance de l'enfant, dans l'Émile, a souvent été ignoré en raison de la fameuse Profession de foi du Vicaire savoyard qu'elles ont pourtant pour fonction d'introduire. En quittant la perspective étroite des études rousseauistes pour adopter la double perspective de la psychologie de la religion et de la philosophie de la religion, cette étude cherche à expliquer et à discuter la thèse de Rousseau selon laquelle l'enfant, faute d'être capable de concevoir l'idée de Dieu, ne croit pas en Dieu mais en ceux qui le lui présentent.

\section{Citer ce document / Cite this document :}

Frey Daniel. La croyance de l'enfant selon Rousseau. Émile à la lumière des philosophie et psychologie de la religion. In: Revue d'histoire et de philosophie religieuses, 95e année $n^{\circ} 3$, Juillet-Septembre 2015. pp. 265-287;

doi : https://doi.org/10.3406/rhpr.2015.1950

https://www.persee.fr/doc/rhpr_0035-2403_2015_num_95_3_1950

Fichier pdf généré le 09/12/2019 


\title{
LA CROYANCE DE L'ENFANT SELON ROUSSEAU
}

\section{Émile à la lumière des philosophie et psychologie de la religion}

\author{
Daniel Frey \\ Faculté de Théologie protestante (EA 4378) - Université de Strasbourg \\ 9 place de l'Université - F-67084 Strasbourg Cedex
}

\begin{abstract}
Résumé : L'intérêt des pages que Rousseau consacre à la croyance de l'enfant, dans l'Émile, a souvent été ignoré en raison de la fameuse Profession de foi $\mathrm{du}$ Vicaire savoyard qu'elles ont pourtant pour fonction d'introduire. En quittant la perspective étroite des études rousseauistes pour adopter la double perspective de la psychologie de la religion et de la philosophie de la religion, cette étude cherche à expliquer et à discuter la thèse de Rousseau selon laquelle l'enfant, faute d'être capable de concevoir l'idée de Dieu, ne croit pas en Dieu mais en ceux qui le lui présentent.
\end{abstract}

Abstract: The interest of the pages which Rousseau devotes in Emile to childhood belief has often been eclipsed by the famous Confession of faith of a Savoyard Vicar, which these pages are supposed to introduce. By leaving behind the narrow perspective of Rousseau Studies and adopting a double perspective of the psychology of religion and philosophy of religion, this article hopes to explain and discuss Rousseau's thesis that the child, incapable of conceiving the idea of God, does not believe so much in God as in the people who present the idea of God.

\section{Croire EN Dieu, CROIRE PIERRE OU JACQUeS : ROUSSEAU, L'ENFANT ET SON DIEU}

Quand un enfant dit qu'il croit en Dieu, ce n'est pas en Dieu qu'il croit, c'est à Pierre ou à Jacques qui lui disent qu'il y a quelque chose qu'on appelle Dieu; et il le croit à la manière d'Euripide :

'Ô Jupiter! Car de toi rien sinon

Je ne connais seulement que le nom, 1

\footnotetext{
${ }^{1}$ Rousseau, 1969 [1762], p. 555 (Rousseau cite Euripide par l'entremise du Traité de l'amour de Plutarque). En attendant la publication des nouvelles œuvres complètes entreprise en 2012 (édition dite du tricentenaire, dans la collection des Classiques Garnier), nous nous référons ici, comme c'est encore l'usage, aux Euvres complètes éditées dans la Bibliothèque de la Pléiade, dont nous modernisons toutefois systématiquement l'orthographe et parfois la ponctuation.
} 
Jean-Jacques Rousseau énonce cette assertion singulière au livre IV de l'Émile, au moment il en arrive enfin à l'épineuse question de l'éducation religieuse qu'il convient de donner à son élève fictif, déjà âgé de 15 ans. Ébauchée selon toute vraisemblance en même temps qu'Émile ${ }^{2}$, la Profession de foi du Vicaire savoyard a été insérée dans cette fiction éducative en lieu et place du chapitre sur l'éducation religieuse. Cette insertion de la Profession de foi $d u$ Vicaire savoyard au livre IV de l'Émile, pour artificielle qu'elle puisse paraître sur le plan formel, ne laisse pas d'être cohérente sur le fond : à un projet d'éducation selon la nature ne pouvait correspondre, en effet, qu'une défense de la religion naturelle, dirigée à la fois contre les philosophes athées et contre les religions prétendument révélées. La Profession de foi du Vicaire savoyard, que Rousseau considérait à juste titre comme sa réflexion la plus aboutie et la plus personnelle sur la religion, ne veut présenter de la religion que ce qui peut être justifié par la raison, même si son objet, par définition, se situe au-delà des limites de la raison humaine. On lit en effet, quelques lignes avant le début de ladite Profession de foi :

C'est surtout en matière de religion que l'opinion triomphe. Mais nous qui prétendons secouer son joug en toute chose, nous qui ne voulons rien donner à l'autorité, nous qui ne voulons rien enseigner à notre Émile qu'il ne pût apprendre de lui-même par tout pays, dans quelle religion l'élèverons-nous ? À quelle secte agrègerons-nous l'homme de la nature ? La réponse est fort simple, ce me semble; nous ne l'agrègerons ni à celle-ci ni à celle-là, mais nous le mettrons en état de choisir celle où le meilleur usage de sa raison doit le conduire ${ }^{3}$.

Cette Profession de foi appelle à honorer Dieu par la raison et la conscience morale qu'il a léguées en partage à tous les hommes. Elle offre, selon son auteur, des " raisons de douter et de croire»: de douter, essentiellement, des révélations dont font état les religions - et de croire en un Dieu universellement accessible par la raison ${ }^{4}$. Dans une étude précédente ${ }^{5}$, il s'agissait de montrer la logique de cette Profession de foi de Rousseau : partant de la dénonciation des conversions extorquées - comme celle qui l'amena lui-même à quitter le protestantisme pour le catholicisme -, elle conduisait son auteur à défendre la religion naturelle, puis à critiquer les religions

\footnotetext{
${ }^{2}$ En effet le premier état de l'Émile (Manuscrit Favre) comporte déjà des passages qui préfigurent directement cette Profession de foi (cf. Rousseau, 1969 [1758-1760], p. 221231), même si des passages de cette dernière remontent à des réflexions encore antérieures (Les Lettres à Sophie [1757], cf. Porset, 1996, p. 753).

${ }^{3}$ Rousseau, 1969 [1762], p. 558 (nous soulignons).

${ }^{4}$ Nous faisons ici allusion au propos final que le Vicaire, masque de Rousseau, adresse à son élève : « Je vous ai dit mes raisons de douter et de croire. Maintenant c'est à vous de juger $;[\ldots]$ Soyez sincère avec vous-même. Appropriez-vous de mes sentiments ce qui vous aura persuadé, rejetez le reste. » (Rousseau, 1969 [1762], p. 630.)

${ }^{5}$ Frey, 2012.
} 
révélées tout en rendant un hommage bien singulier à l'Évangile, pour finalement appeler le lecteur à demeurer dans la religion de ses pères. Nous nous proposons ici de remonter le fil du discours de Rousseau en amont de la Profession de foi, de manière à considérer ses diverses analyses et prises de positions relatives à l'enfant et à sa croyance en Dieu, illustrées par le propos cité en exergue.

\section{UNE PERSPECTIVE NOUVELLE : \\ LA FICTION PÉDAGOGIQUE DE ROUSSEAU \\ À LA LUMIĖRE DE LA PHILOSOPHIE DE LA RELIGION \\ ET DE LA PSYCHOLOGIE DE LA RELIGION}

Aussi surprenant que cela puisse paraître s'agissant d'un auteur autant commenté que Jean-Jacques Rousseau, les considérations de ce dernier sur la croyance de l'enfant n'ont pas reçu l'attention qu'elles méritent. Le caractère systématique de la Profession de foi a sans aucun doute masqué l'intérêt des propos sur la religion qui émaillent les livres I à IV de l'Émile. Le silence de la plupart des commentateurs sur ces derniers est frappant dans deux récentes entreprises collectives destinées à réévaluer aujourd'hui le projet pédagogique de Rousseau, à l'occasion du tricentenaire de sa naissance et du $250^{\mathrm{e}}$ anniversaire de la publication de l'Émile ${ }^{6}$. Sur les cinquante études que rassemblent ces collectifs, seules deux études abordent la question de la religion. La première ne s'intéresse pas au propos de Rousseau précédant la Profession de foi, alors même que son auteur prête la plus grande attention à la façon dont cette dernière participe à l'économie de l'Émile ${ }^{7}$. Seule la seconde évoque, trop brièvement, le jugement du philosophe à l'endroit de la croyance chez l'enfant, mais là encore sans analyser ce jugement en lui-même ${ }^{8}$. Ce quasi silence des commentateurs, s'il est surprenant, n'est pas inexplicable : soit c'est la cohérence de la pensée de Rousseau en matière de religion que questionnent les commentateurs - et dans ce cas il est compréhensible, encore une fois, que ce soit la Profession de foi qui fasse l'objet de toutes les attentions; soit c'est la question de la valeur de la pédagogie rousseauiste qui est posée - et dans ce cas les pédagogues et philosophes de l'éducation ne se sentent pas concernés pas la question de l'enseignement religieux à donner à Émile.

Pour prolonger notre remarque, reprenons séparément ces deux types de préoccupations. Au sujet de la première, disons sans ambages

\footnotetext{
${ }^{6}$ Voir Habib, 2012 ; Drouin-Hans, 2013.

${ }^{7}$ Bernardi, 2012.

${ }^{8}$ Lamarre, 2013.
} 
que la recherche de la cohérence des écrits de Rousseau en matière de religion occulte trop souvent l'intérêt qu'il y a à penser ce que Rousseau thématise expressément dans ses essais. D'une façon générale, les études rousseauistes s'évertuent, et en un sens s'épuisent un peu vainement, à dire la cohérence de la pensée de Rousseau, plutôt qu'à penser à partir de Rousseau. On ne niera pas que ce travail soit inévitable dans la mesure où les différents régimes discursifs (autobiographie, autofiction, traités, lettres-manifestes, etc.) mis en œuvre par cet auteur éminemment polygraphe supposent des traitements différents, et amènent ainsi à s'interroger sur l'improbable synthèse de cette pensée ${ }^{9}$. Cette tâche est léguée par Rousseau lui-même : conscient et même créateur des paradoxes de sa personnalité, il s'est sans cesse appliqué à déterminer ce qui le singularisait parmi ses contemporains et le poussait à s'ouvrir à eux et à se faire comprendre d'eux. Ce n'est pas un moindre paradoxe que pour penser la croyance avec Rousseau, il faille sortir du souci de la cohérence de son œuvre ! Peu importera ici, en conséquence, la cohérence réelle ou supposée de la pensée de Rousseau en matière de religion : c'est de ses thèses sur la croyance de l'enfant qu'il s'agira, dans la perspective d'une philosophie de la religion qui a précisément élevé le concept de croyance au rang de ses catégories conceptuelles principales, comme l'attestent à l'envi les récentes productions dans ce champ ${ }^{10}$.

Mais ce choix de relire Émile dans la perspective de la philosophie de la religion, dont on vient de dire les avantages, est nécessaire mais non suffisant, dans la mesure où les thèses rousseauistes quant à la croyance de l'enfant demandent de prendre en compte la description du développement psychologique de l'enfant qui les fonde. Il convient dès lors de préciser la perspective choisie ici, en associant philosophie de la religion et psychologie de la religion. Certes, on conviendra que celles-ci, en tant que disciplines spécifiques, sont postérieures à l'œuvre de Rousseau : Jean Greisch, auteur

\footnotetext{
${ }^{9}$ Insistons sur le fait que ces traitements sont très divers, selon qu'il s'agit de passages de romans (dans La Nouvelle Héloïse), de fragments personnels (par exemple ses deux prières : Rousseau, 1969, p. 1033-1039), de lettres philosophiques ou d'un essai synthétique comme la Profession de foi, d'attaques contre les représentants du christianisme, catholiques ou protestants, etc. Si d'anciennes études les mêlaient indûment (par exemple Guyot, 1962 ; plus récemment Rosenberg, 1996), d'autres tiennent heureusement à les distinguer, comme celle de Bernardi, 2012.

${ }^{10}$ C'est essentiellement aux essais relevant de la philosophie analytique de la religion que l'on doit cette attention au concept de croyance (voir Bourgeois-Gironde, 2002, Pouivet, 2003, Pouivet, 2006 [dans la présente Revue], Bouveresse, 2007, Michon - Pouivet, 2010), mais des auteurs comme Ricœur (Ricœur, 2000 [1969]) ont pu également lui consacrer d'importants textes. Toutefois, si la philosophie de la religion d'inspiration anglo-saxonne s'intéresse beaucoup à la croyance, elle le fait sans égard pour l'apport de Rousseau en la matière. On notera d'ailleurs qu'au sein de la philosophie de la religion d'inspiration herméneutique, même l'imposante trilogie de Jean Greisch (Greisch, 2002), pourtant riche en analyses portant sur des œuvres antérieures à l'apparition de la philosophie de la religion, ne croit pas utile de consacrer quelques pages à la pensée de Rousseau en la matière.
} 
d'une remarquable trilogie consacrée à la philosophie de la religion, fait remonter la première aux Discours sur la religion [1799] de Schleiermacher ${ }^{11}$, et l'on sait que la seconde reconnaît dans l'ouvrage de William James intitulé L'expérience religieuse. Essai de psychologie descriptive [1902] un ouvrage fondateur ${ }^{12}$. Mais cela ne saurait empêcher qu'une œuvre soit lue à leur lumière, surtout si elle anticipe certaines de leurs préoccupations. Or c'est bien le cas de l'Émile, où Rousseau entend appuyer son refus de toute éducation religieuse sur une observation attentive de l'enfant donnant lieu à une esquisse de description des différentes étapes de son développement psychologique. L'ambition descriptive est manifeste dès le début de l'ouvrage, quand bien même le qualificatif «psychologique » n'est pas employé, son usage courant remontant au XIX ${ }^{\mathrm{e}}$ siècle :

On ne connaît point l'enfance : sur les fausses idées qu'on en a, plus on va, plus on s'égare. Les plus sages s'attachent à ce qu'il importe aux hommes de savoir, sans considérer ce que les enfants sont en état d'apprendre. Ils cherchent toujours l'homme dans l'enfant, sans penser à ce qu'il est avant que d'être homme. Voilà l'étude à laquelle je me suis le plus appliqué, afin que, quand toute ma méthode serait chimérique et fausse, on pût toujours profiter de mes observations. Je puis avoir très mal vu ce qu'il faut faire, mais je crois avoir bien vu le sujet sur lequel on doit opérer. Commencez donc par mieux étudier vos élèves ; car très assurément vous ne les connaissez point. Or si vous lisez dans cette vue, je ne le crois pas sans utilité pour vous ${ }^{13}$.

Un autre passage, extrait de ses Confessions, fonde directement sa décision de ne point parler de religion à Émile sur son observation de l'enfant, et non sur sa propre expérience, lui-même ayant reçu une éducation religieuse qu'il juge à bien des égards exemplaire et qui ne l'a pas détourné de la foi ; Rousseau, notons-le en passant, n'a jamais perdu une occasion de dire à ses anciens amis philosophes que le sentiment religieux a toujours été pour lui une évidence. Voici ce passage, dont le contexte ${ }^{14}$, sans être inintéressant, n'est pas ici déterminant :

\footnotetext{
${ }^{11}$ Greisch, 2002, p. 77.

${ }^{12}$ James, 1931 [1902]. Ajoutons que c'est la psychologie de la religion, et non plus la pédagogie, qui se préoccupe aujourd'hui des représentations que se font les enfants du divin.

13 «Préface » de l'Émile: Rousseau, 1969 [1762], p. 241-242.

${ }^{14} \mathrm{Au}$ Livre II des Confessions, Rousseau décrivait son enfance auprès de son père, genevois sincèrement croyant, puis, au moment d'aborder son passage au catholicisme pour l'expliquer et le faire bien comprendre, il revient sur sa première éducation religieuse : "Mon père, quoique homme de plaisir, avait non seulement une probité sûre, mais beaucoup de religion. Galant homme dans le monde et chrétien dans l'intérieur, il m'avait inspiré de bonne heure les sentiments dont il était pénétré. » (Rousseau, 1959 [1769], p. 61.) Plus loin, au sujet de l'éducation donnée (en l'absence de son père) par le pasteur Lambercier, il ajoute : " sa sœur et lui cultivèrent, par des instructions douces et judicieuses, les principes de piété qu'ils trouvèrent dans mon cœur. Ces dignes gens employèrent pour cela des moyens si vrais, si discrets, si raisonnables, que, loin de m'ennuyer au sermon, je n'en sortais jamais sans être intérieurement touché et sans faire des résolutions de bien vivre, auxquelles je manquais rarement en y pensant. » (Rousseau, 1959 [1769], p. 62.)
} 
J'avais donc de la religion tout ce qu'un enfant à l'âge où j'étais en pouvait avoir. J'en avais même davantage, car pourquoi déguiser ici ma pensée ? Mon enfance ne fut point d'un enfant; je sentis, je pensai toujours en homme. Ce n'est qu'en grandissant que je suis rentré dans la classe ordinaire ; en naissant, j'en étais sorti. [...]. Ainsi, quand j'ai dit qu'il ne fallait point parler aux enfants de religion si l'on voulait qu'un jour ils en eussent, et qu'ils étaient incapables de connaître Dieu, même à notre manière, j'ai tiré mon sentiment de mes observations, non de ma propre expérience : je savais qu'elle ne concluait rien pour les autres. Trouvez des Jean-Jacques Rousseau à six ans, et parlez-leur de Dieu à sept, je vous réponds que vous ne courez aucun risque ${ }^{15}$.

Avant de discuter de ces observations relatives à la croyance de l'enfant, et pour être en état de le faire, reconnaissons que les observations dont Rousseau fait état, non sans fierté, ont en effet contribué à faire de lui un précurseur, sinon de la psychologie de l'enfant au sens strict du terme, du moins de son souci de ne pas interpréter les comportements enfantins et leurs développements à l'aune de l'état adulte. Rousseau pose en effet pour principe de respecter la singularité de l'enfance, à la fois comme un état ayant sa valeur propre et son bonheur, et comme une succession de phases dont chacune constitue une forme d'équilibre. Ce principe, que résume joliment 1'heureuse formule appelant à « laisser mûrir 1'enfance dans les enfants ${ }^{16} »$, a préparé les tentatives de schématisation des différents stades du développement de l'enfant établis par la psychologie de l'enfant.

Celle-ci ne s'est d'ailleurs pas fait faute d'interroger les observations et propositions contenues dans l'Émile. À la question de savoir si Rousseau s'est montré le précurseur de la psychologie de l'enfant, les psychologues eux-mêmes donnèrent toutefois des réponses contrastées. Si Édouard Claparède (1873-1940) et Henri Wallon (1879-1962) ont salué la naissance de leur science dans l'Émile ${ }^{17}$, Jean Piaget (1896-1980), fondateur de la psychologie génétique ${ }^{18}$, a estimé (au contraire de son maître Claparède) que si « Rousseau a entrevu que 'chaque âge a ses ressorts', que 'l'enfant a des manières de voir, de penser et de sentir qui lui sont propres', [...] ces idées sont restées à l'état de 'croyance sociologique', voire d' 'instrument

\footnotetext{
${ }^{15}$ Rousseau, 1959 [1769], p. 62. Vargas (2012, p. 151) explique que si Rousseau fait exception à son propre programme, c'est simplement parce qu'il existe, dans la nature, des «monstres »: en l'occurrence, « Rousseau est un monstre positif, car il est en avance sur le rythme de la nature ».

${ }^{16}$ Rousseau, 1969 [1762], p. 324.

${ }^{17}$ Cf. Loeffel, 2013, p. 86-88.

${ }^{18}$ L'ouvrage classique en la matière est le « Que sais-je ? » rédigé par Piaget et Inhelder, La psychologie de l'enfant (Piaget - Inhelder, 2003 [1966]). Il est aujourd'hui remplacé dans la célèbre collection par un ouvrage d'Olivier Houdé (Houdé, 2013), signe manifeste d'une évolution de la psychologie de l'enfant depuis Piaget, même si l'auteur lui consacre encore tout un chapitre.
} 
polémique', faute d'être étayées par l'observation objective de l'expérience ${ }^{19}$ ». Selon Piaget, non seulement Rousseau a cru pouvoir fixer arbitrairement les stades du développement de l'enfant, sans se soucier du passage de l'un à l'autre (ces stades constituant en fait une suite de tableaux), mais il a eu le grand tort de considérer le développement de l'enfant en dehors de son environnement social. Piaget a bien vu que la description, dans l'Émile, n'est pas seulement vouée à déterminer ce qu'est l'enfant à chaque stade de sa croissance. Elle est aussi destinée à prescrire ce que doit être le développement d'un enfant qui, idéalement et fictivement, échapperait tout à fait à l'emprise des préjugés et des artifices de la société, et serait éduqué conformément au développement naturel de ses sens et de son intelligence.

Rousseau est en effet convaincu que toute réalité naturelle est bonne en soi, à commencer par la nature humaine, pervertie par la vie de l'homme en société ${ }^{20}$. Les premiers mots du Livre I de l'Émile le proclament avec une force qui doit ici être rappelée : "Tout est bien, sortant des mains de l'Auteur du monde : tout dégénère entre les mains de l'homme ${ }^{21}$. " L'éducation a toujours été, jusqu'ici, un artifice contre-nature, en ceci qu'elle a systématiquement fait primer les préjugés des conventions sociales sur le développement naturel de l'enfant. Rousseau entend redonner à ce dernier la préséance, tout simplement parce qu'il est le seul inévitable : "l'éducation par la nature » vient toujours avant «l'éducation des choses» et "l'éducation des hommes ${ }^{22}$ ». Si le Genevois s'en tenait là, il serait indubitablement le précurseur de la psychologie de l'enfant. Or il ne cherche pas seulement à dire quels sont les stades naturels de la croissance de 1 'enfant ${ }^{23}$. Il cherche surtout à énoncer les principes d'une éducation naturelle suivant pas à pas le lent développement des facultés de l'enfant, au rythme de la nature et non à celui, toujours trop rapide, de l'éducation des hommes. L'éducation que théorise et

${ }^{19}$ Loeffel, 2013, p. 91. Les propos entre guillemets sont ceux de Piaget [« Les méthodes nouvelles. Leurs bases psychologiques », 1935], eux-mêmes en référence à Rousseau : «L'enfance a des manières de voir, de penser, de sentir qui lui sont propres ; rien n'est moins sensé que d'y vouloir substituer les nôtres » (Rousseau, 1969 [1762], p. 319).

${ }^{20}$ Cf. Frey, 2012, p. 154.

${ }^{21}$ Rousseau, 1969 [1762], p. 245.

${ }^{22}$ Rousseau, 1969 [1762], p. 247.

${ }^{23}$ C'est là l'objectif de la psychologie de l'enfant moderne, cf. Piaget - Inhelder, 2003 [1966], p. 3 : «La psychologie de l'enfant étudie la croissance mentale ou, ce qui revient au même, le développement des conduites (c'est-à-dire des comportements y compris la conscience), jusqu'à cette phase de transition constituée par l'adolescence, qui marque l'insertion de l'individu dans la société adulte. [...] La croissance mentale est indissociable de la croissance physique, notamment de la maturation des systèmes nerveux et endocriniens, se poursuivant jusque vers 16 ans » (les auteurs soulignent). 
prône Rousseau est donc « négative ${ }^{24}$ ». Pour laisser l'enfant suivre " la marche naturelle du cœur humain », il y a beaucoup à faire : " empêcher que rien ne soit fait ${ }^{25}$ ", c'est-à-dire, non pas empêcher une éducation par les hommes - projet absurde puisque Rousseau, le tout premier, affirme que l'être humain doit par nature être élevé ${ }^{26}$ mais écarter de l'enfant tout enseignement (toute connaissance, toute pratique) qui ne proviendrait pas de l'expérience qu'il peut faire, lui-même, des capacités et des jugements qu'il acquiert progressivement. L'homme qui aura pour rôle d'appliquer cette pédagogie théorique et normative - cette expérience de la pensée, en soi fictive - n'est autre que le précepteur d'Émile. Par bien des traits, il est une figure (une de plus) de Rousseau lui-même. Cet éducateur idéal sera chargé, durant vingt années, de protéger son élève de l'influence des préjugés de la société ${ }^{27}$, à la place du père de l'enfant dès le sevrage ${ }^{28}$. Ce n'est pas le lieu, ici, de s'attarder sur cette figure et le dispositif précis qui l'accompagne. Il suffira de noter que le précepteur va diriger toute l'éducation naturelle d'Émile, non par ses paroles ( « Ne donnez à votre élève aucune espèce de leçon verbale, il n'en doit recevoir que de l'expérience $\left.{ }^{29} »\right)$, mais par

24 « La première éducation doit donc être purement négative. Elle consiste, non point à enseigner la vertu ni la vérité, mais à garantir le cœur du vice et l'esprit de l'erreur. » (Rousseau, 1969 [1762], p. 323.)

${ }^{25}$ Rousseau, 1969 [1762], p. 265, p. 251 pour les deux citations.

${ }^{26}$ Voir Rousseau, 1969 [1762], p. 247 : «On façonne les plantes par la culture, et les hommes par l'éducation. Si l'homme naissait grand et fort, sa taille et sa force lui seraient inutiles jusqu'à ce qu'il eût appris à s'en servir; elles lui seraient préjudiciables, en empêchant les autres de songer à l'assister; et, abandonné à lui-même, il mourrait de misère avant d'avoir connu ses besoins. [...] Tout ce que nous n'avons pas à notre naissance et dont nous avons besoin étant grands, nous est donné par l'éducation. »

${ }^{27}$ Signalons en passant que pour Rousseau partage avec d'autres philosophes des Lumières son opposition à l'éducation des collèges jésuites et à sa " surveillance ecclésiastique » (Hager, 2007 [1997], p. 430). L'éducation d'Émile sera privée, puisque les collèges sont trop liés à l'Église et que l'éducation publique ne peut plus exister dans un monde où selon lui les notions de patrie et de citoyen ne signifient plus rien, contrairement au monde antique : cf. L'Aminot, 1996b, p. 282.

${ }^{28}$ Sur ce sujet, Rousseau, 1969 [1762], p. 262s. Selon Marie-France Morel (Morel, 2007), si le siècle des Lumières est bien celui de la "découverte de l'enfance " et de l'importance du rôle des parents, il est aussi celui où le recours aux nourrices a été le plus grand. Son propos sur le rôle paternel, attribué ici au précepteur, est l'occasion pour Rousseau de dire son remord d'avoir mis ses cinq enfants aux Enfants trouvés. N'oublions pas, face à ce sujet inévitable dès qu'il s'agit d'Émile, la grande fréquence des abandons d'enfants sous l'Ancien Régime : il y eut à peu près 25000 abandons d'enfant en France par an, au point que des institutions spécifiques furent créées (par exemple par saint Vincent de Paul). Ces institutions où l'État supplée à la responsabilité familiale furent d'ailleurs l'occasion d'un débat en Europe : constitueraient-elles un encouragement à délaisser les enfants ? (Morel, 2007, p. 461s.) Rousseau, en abandonnant ses enfants au motif de son indigence, est donc - soit dit sans l'excuser - loin d'être le seul parmi les couches modestes de la société. Et que dire de l'exemple de son père se déchargeant de ses devoirs en fuyant Genève et en confiant son fils ensuite à d'autres ?

${ }^{29}$ Rousseau, 1969 [1762], p. 321. Cf. p. 447 : «Les choses, les choses ! Je ne répéterai jamais assez que nous donnons trop de pouvoir aux mots : avec notre éducation babillarde nous ne faisons que des babillards. ") 
ses stratagèmes destinés à le placer dans diverses circonstances où, sans jamais commenter ni même dire ce qu'il fait, il va procéder de sorte que le développement naturel de l'élève ait lieu. En cela, il sera un bon « ministre de la nature ${ }^{30} »$.

L'erreur de Rousseau que soulignait Piaget (ne pas considérer l'enfant dans son milieu social, et surtout ne pas placer Émile au sein de sa famille, ni parmi les enfants de son âge) ne tient donc pas tant au fait que le philosophe ne considérait pas la croissance mentale de l'enfant à travers ses interactions (car l'éducation demeure pour Rousseau un accompagnement sollicitant l'enfant) qu'au fait de vouloir priver l'enfant des sollicitations extérieures, dans le cercle des proches et dans celui des enfants, lui dictant ainsi des interactions conçues en fonction du développement théorique d'un enfant élevé en vase clos. Comme le dit fort justement Jean-François Perrin, « l'enfant Émile est la modélisation d'une hypothèse sur l'enfance à l'état nature ${ }^{31} »$. L'élaboration de cette hypothèse relève d'une théorie anthropologique comportant une dimension politique dont il ne sera pas question ici ${ }^{32}$, dans la mesure où nous prenons seulement pour objet les observations et positions de Rousseau relatives à la croyance de l'enfant. Le choix de notre double perspective (psychologie de la religion et philosophie de la religion) se justifie précisément par le fait que, pour comprendre ce que Rousseau affirme du rapport de l'enfant à la croyance, il est nécessaire de prêter attention à deux types d'affirmations, les unes relevant de sa description du développement psychologique de l'enfant, les autres de sa définition de la croyance comme complément de la connaissance. Distinctes en théorie, ces affirmations se voient souvent superposées sous sa plume. A des fins didactiques, nous allons ici prêter une attention séparée à ces deux types d'assertions, dans la perspective de la psychologie de la religion tout d'abord, puis dans celle de la philosophie de la religion.

\footnotetext{
${ }^{30}$ Rousseau, 1969 [1762], p. 639. Pour nous, lecteurs du XXI ${ }^{\mathrm{e}}$ siècle, son rôle est quasi démiurgique : le précepteur guide chaque pas de son élève à son insu; il entend contrôler tout ce qui le touche, et paraît même d'autant plus manipulateur qu'il cache son emprise et sa volonté toute-puissante derrière le « hasard » des rencontres et des situations. Il n'est que trop évident (voir par exemple Rousseau, 1969 [1762], p. 363) que Rousseau n'aurait pas du tout compris nos réticences envers ce procédé qui tient seulement pour lui de la fiction éducative.

${ }^{31}$ Perrin, 2012, p. 50.

${ }^{32}$ Émile forme en effet un diptyque avec le Contrat social paru simultanément. Notons seulement que c'est en se figurant l'éducation d'un homme qui serait, pour la première fois, élevé pour lui-même et non pour les autres - un homme qui du coup serait authentiquement lui-même - que Rousseau croit pouvoir faire œuvre politique : seul un tel homme, assuré de son être, soucieux de son rôle social sans se confondre avec lui, pourra véritablement être bon et être social. Comme l'écrit très justement Y. Vargas (cité dans L'Aminot, 1996a, p. 278) : Rousseau «ne propose pas d'éduquer un enfant au sein d'une société, mais de construire une nouvelle société au sein du développement d'un homme».
} 


\section{LA PSYCHOLOGIE DU DÉVELOPPEMENT RELIGIEUX DE L'ENFANT SELON ROUSSEAU}

Nous avons tous été enfants, mais avons tous oublié, faute d'avoir pu à ce moment le thématiser - et surtout faute de s'en être soucié le moins du monde ! - ce qu'est penser en enfant. Le psychologue de l'enfance s'évertue à déterminer ce qu'est cette pensée, en créant des exercices d'observation lui permettant de reconstruire la logique de l'enfant, à chaque stade de sa vie. De l'existence même des procédures d'analyse et d'objectivation des conduites cognitives de l'enfant, il découle pour le psychologue que le langage, loin de constituer la source de la logique, « est au contraire structuré par elle ${ }^{33}$ ", et que la phase d'acquisition du langage ne signifie pas qu'il existe une identité entre ce qu'entendent les adultes et les enfants par les mêmes mots ${ }^{34}$. Tout en étant fort loin de la sophistication de la psychologie moderne, Rousseau observe déjà cette distorsion entre notre usage des mots et celui des enfants. Le principe d'éducation négative que nous avons décrit repose sur cette observation, particulièrement décisive, à ses yeux : la pensée de l'enfant n'obéit pas à la même logique que la nôtre, et s'il emploie les mêmes mots que nous, ce n'est pas pour leur donner le même sens. Rousseau notait ainsi :

Les pensées les plus brillantes peuvent tomber dans le cerveau des enfants ou plutôt les meilleurs mots dans leur bouche comme les diamants du plus grand prix sous leurs mains sans que pour cela ni les pensées ni les diamants leur appartiennent ; [...] Les choses que dit un enfant ne sont pas pour lui ce qu'elles sont pour nous, il n'y joint pas les mêmes idées. Ces idées, si tant est qu'il en ait, n'ont dans sa tête ni suite ni liaison; rien de fixe, rien d'assuré dans tout ce qu'il pense. Examinez votre prétendu prodige. En de certains moments vous lui trouverez un ressort d'une extrême activité, une clarté d'esprit à percer les nues. Le plus souvent ce même esprit vous paraît lâche, moite, et comme environné d'un épais brouillard. Tantôt il vous devance et tantôt il reste immobile. Un instant vous direz: 'c'est un génie', et l'instant d'après 'c'est un sot'; vous vous tromperiez toujours : c'est un enfant ${ }^{35}$.

C'est cette observation, répétée à plusieurs reprises à différents âges de l'enfant, qui inspire à Rousseau le vœu de retarder toute

\footnotetext{
${ }^{33}$ Piaget - Inhelder, 2003, [1966], p. 69.

${ }^{34}$ Piaget - Inhelder interrogent par exemple le jeu symbolique d'une fillette de deux ans affirmant «Je suis le canard mort! » (2003, [1966], p. 45s), et se montrent soucieux eux aussi de ne pas tenir l'équivalence des termes employés par les enfants (à un certain stade de leur développement cognitif) avec l'équivalence des idées que nous, nous lions à eux : «Le signe [...], étant conventionnel, est nécessairement collectif : l'enfant le reçoit donc, par le canal de l'imitation [...]; seulement il le façonne aussitôt à sa manière » (p. 43).

${ }^{35}$ Rousseau, 1969 [1762], p. 342 (ponctuation modifiée). À de tels propos, et malgré bien des limites, on voit à quel point Rousseau a été désireux, comme nul avant lui, de saisir la singularité de l'enfant, en ne cherchant pas déjà en lui l'homme qu'il sera.
} 
leçon qui ne lui semble pas nécessaire à sa survie, afin qu'il juge seulement de ce qu'il est en état de comprendre ${ }^{36}$. Les enfants savent raisonner, et raisonnent d'ailleurs très bien dans les choses dont ils ont une utilité immédiate ${ }^{37}$. Mais trop souvent, dit Rousseau, l'on attend d'eux qu'ils acquiescent à des réalités dont l'intérêt leur échappe à cette heure. On leur rend pénibles des bienfaits en les leur présentant comme des devoirs. Ce qui est vrai de la moralité l'est aussi de la piété, et c'est paradoxalement en raison du prix que Rousseau leur accorde qu'il appelle à ne pas les présenter trop tôt, ni trop formellement :

Pour paraître leur prêcher la vertu, on leur fait aimer tous les vices. On les leur donne en leur défendant de les avoir. Veut-on les rendre pieux ? On les mène s'ennuyer à l'église ; en leur faisant incessamment marmotter des prières, on les force d'aspirer au bonheur de ne plus prier Dieu $^{38}$.

Le lecteur d'aujourd'hui ne doit pas trop vite glisser sur un tel propos, car ne pas donner d'éducation religieuse à l'enfant, ni le conduire à l'église (comme c'est le cas, fictivement, pour Émile) relève au XVIII ${ }^{\mathrm{e}}$ siècle du non-conformisme le plus affirmé. Émile n'a pas suivi dès son plus jeune âge le catéchisme, il n'a jamais fréquenté d'église, et n'a pas la plus élémentaire connaissance des dogmes et des pratiques chrétiennes. Or l'éducation que les pédagogues théorisaient à cette époque avait pour cadre le christianisme et pour toile de fond le péché originel ${ }^{39}$. À l'Archevêque de Beaumont condamnant l'Émile et l'éducation que Rousseau y théorise, ce dernier écrira en 1763 :

J'ai prouvé que cette éducation [religieuse et traditionnelle], que vous appelez la plus saine, était la plus insensée, que cette éducation, que vous appelez la plus vertueuse, donnait aux enfants tous les vices ; j'ai prouvé que toute la gloire du paradis les tentait moins qu'un morceau de sucre, et qu'ils craignaient beaucoup plus de s'ennuyer à Vêpres que de brûler en enfer ${ }^{40}$.

Le propos, par delà l'excès rhétorique, en confirme d'autres qui montrent que le vrai danger pour Rousseau n'est pas de laisser

\footnotetext{
${ }^{36}$ Cf. Rousseau, 1969 [1762], p. 324. Signalons ici qu'une très belle raison de laisser à l'enfant le temps de son développement est de lui donner de vivre pleinement cette étape de sa vie, qui bien souvent risque d'être la dernière : un enfant sur quatre n'atteindra jamais sa première année et un sur deux, seulement, arrivera à l'âge adulte (voir Morel, 2007, p. 459). Une vie aussi incertaine ne saurait pour Rousseau perdre des occasions de bonheur : « Vous êtes alarmés de le voir consumer ses premières années à ne rien faire ! Comment ! N'est-ce rien que d'être heureux ? N'est-ce rien que de sauter, jouer, courir toute la journée ? De sa vie il ne sera si occupé. » (Rousseau, 1969 [1762], p. 343-344.)

${ }^{37}$ Cf. Rousseau, 1969 [1762], p. 345.

${ }^{38}$ Rousseau, 1969 [1762], p. 338. « Marmotter » est une variante de « marmonner».

${ }^{39}$ Voir Sermain, 2012.

${ }^{40}$ Rousseau, 1969 [1763], p. 943.
} 
l'enfant dans l'ignorance du religieux, mais de le conduire à en profaner le sacré. Il est plus grave qu'on ne le pense d'emmener des enfants s'ennuyer à l'église. L'absence d'éducation relative au Dieu des chrétiens paraît à tout prendre moins dommageable pour Rousseau que ces premières impressions négatives qui pourraient bien tenir les enfants définitivement éloignés du divin. Pour le reste, et il le redira face à l'Archevêque, Rousseau craint d'autant moins de laisser Émile dans l'ignorance de ce qu'est le péché que lui-même combat le dogme du péché originel et affirme avec conviction la bonté naturelle de l'homme ${ }^{41}$. Moralité et piété ne sont pensables et possibles - et donc également exigibles - aux yeux de Rousseau qu'à partir de l'adolescence, vers quinze ans, lorsque la raison est développée et que la conscience morale, distincte de la raison, peut s'appuyer sur cette dernière. Cet âge correspond précisément pour Rousseau à celui des passions, qui sont potentiellement destructrices sur le plan moral, mais peuvent alors être canalisées ; alors que le jeune enfant, s'il peut mal faire, n'a pas encore conscience de faire le mal $^{42}$. Il n'est donc aucunement fortuit que le stade de l'adolescence soit celui de l'éducation religieuse et de l'éducation morale : les deux supposent une raison constituée, et le retard de l'une comme de l'autre se justifie par la volonté de ne pas imposer d'autorité à l'enfant la religion et la morale, mais d'attendre que, devenu adolescent et ayant donc atteint les portes de la société des adultes, il soit capable d'en percevoir par lui-même la nécessité ${ }^{43}$.

C'est une conviction forte chez Rousseau, en effet, que «ce que Dieu veut qu'un homme fasse, il ne le lui fait pas dire par un autre homme, il le lui dit lui-même, il l'écrit au fond de son cœur ${ }^{44}$ ". Dieu se laisse connaître et fait connaître sa volonté par la raison et la conscience. Discourir sur Dieu devant l'enfant, lui apporter un enseignement religieux plutôt que d'attendre qu'il soit en état de le découvrir de lui-même n'est pas seulement contraire à la psychologie de l'enfant, d'un point de vue cognitif, mais c'est également contraire à la piété, car à exposer l'enfant à ne connaître et à ne dire de Dieu que ce qu'il aura répété sans le comprendre, on l'expose au danger de ne jamais rien savoir de Dieu par lui-même, et à se détourner de lui dès que l'inanité des croyances, apprises mais non comprises, lui apparaîtra. En un mot, au sujet de Dieu et même de

\footnotetext{
${ }^{41}$ Au point d'ailleurs d'affirmer dans Rousseau Juge de Jean-Jacques que son «Émile [...] n'est qu'un traité de la bonté originelle de l'homme » (Rousseau, 1959 [1773-1774], p. 934).

${ }^{42}$ Cf. Rousseau, 1969 [1762], p. 288.

${ }^{43}$ Il est singulier, toutefois, que l'exemple de la piété ne soit pas présenté à Émile alors même qu'il a tant de prix aux yeux de Rousseau.

${ }^{44}$ Rousseau, 1969 [1762], p. 491. Rousseau évoque ici la conscience morale, qui est la voix même de Dieu (voir Frey, 2012, p. 165-166).
} 
l'existence de l'âme, tout « ce qu'il apprend plus tôt qu'il ne faut, il court le risque de ne le savoir jamais ${ }^{45} »$ : avant l'âge de raison, il se fera nécessairement de Dieu une vision que Rousseau n'hésite pas à qualifier d' « idolâtre ${ }^{46} »$.

Il ne sert à rien, par conséquent, d'énoncer devant les enfants des vérités hors d'atteinte. Toute la pédagogie rousseauiste constitue un effort pour arracher l'éducation aux facilités du langage : il ne coûte rien de parler, cela se fait sans peine et peu d'esprits se piquent de ne dire que ce qu'ils ont réellement expérimenté. De là provient notamment la critique radicale du savoir livresque présenté à l'enfant ${ }^{47}$. S'agissant de l'existence de Dieu et des qualités divines, il importe donc avant tout de ne pas « nous payer de mots ${ }^{48}$ » et, pour ce faire, de limiter les croyances à leur plus simple et plus rationnelle expression. C'est là que la psychologie de l'enfant, même naissante, croise la philosophie de la religion, attentive à la justification (et donc la limitation) des croyances. Si déjà les adultes savent à peine ce que signifient les notions relatives à l'Être divin, à combien plus forte raison les enfants seront-ils, pour Rousseau, hors d'état de les appréhender :

L'Être incompréhensible qui embrasse tout, qui donne le mouvement au monde et forme tout le système des êtres, n'est ni visible à nos yeux, ni palpable à nos mains; il échappe à tous nos sens : l'ouvrage se montre, mais l'ouvrier se cache. Ce n'est pas une petite affaire de connaître enfin qu'il existe, et quand nous sommes parvenus là, quand nous nous demandons : quel est-il ? Où est-il ? Notre esprit se confond, s'égare, et nous ne savons plus que penser. [...] Les idées de création, d'annihilation, d'ubiquité, d'éternité, de toute puissance, celle des attributs divins, toutes ces idées qu'il appartient à si peu d'hommes de voir aussi confuses qu'elles le sont, et qui n'ont rien d'obscur pour le peuple parce qu'il n'y comprend rien du tout, comment se représenteront-elles $[\ldots]$ à de jeunes esprits encore occupés aux premières opérations des sens, et qui ne conçoivent que ce qu'ils touchent? C'est en vain que les abîmes de l'infini sont ouverts tout autour de nous ; un enfant n'en sait point être épouvanté ; ses faibles yeux n'en peuvent sonder la profondeur. Tout est infini pour les enfants ; [...] Ils estimeront un espace immense bien plus par leurs pieds que par leurs yeux [...] Si on leur parle de la puissance de Dieu, ils l'estimeront presque aussi fort que leur père ${ }^{49}$.

\footnotetext{
${ }^{45}$ Rousseau, 1969 [1762], p. 554.

${ }^{46}$ Rousseau, 1969 [1762], p. 553 : « Tout enfant qui croit en Dieu est [...] nécessairement idolâtre, ou du moins anthropomorphite ». Cf. p. 556: « Le grand mal des images difformes de la divinité qu'on trace dans l'esprit des enfants est qu'elles y restent toute leur vie, et qu'ils ne conçoivent plus étant hommes d'autre Dieu que celui des enfants. » Sur ce sujet, cf. Burgelin, 1962, p. 14 et 20.

${ }^{47}$ Nous l'avions abordée dans notre étude précédente : Frey, 2012, p. 170-173.

${ }^{48}$ Rousseau, 1969 [1762], p. 293. L'expression est récurrente chez Rousseau.

${ }^{49}$ Rousseau, 1969 [1762], p. 551 et 553.
} 
La comparaison chez l'enfant entre le pouvoir de Dieu et celui de son père, loin de n'être qu'un propos plaisant sous la plume de Rousseau, relève d'une observation que les psychologues de la religion font encore aujourd'hui chez les enfants en âge préscolaire ${ }^{50}$. Plus généralement, du point de vue de la psychologie de la religion actuelle, il est établi que le développement religieux de l'enfant ne peut être appréhendé qu'en fonction de son développement cognitif ${ }^{51}$. Même s'il est loin de présenter ce dernier avec la rigueur de la psychologie moderne, Rousseau rapproche l'idée d'un Être divin invisible et l'enseignement traditionnel le concernant (éternité, ubiquité, etc.) des capacités d'abstraction réelles de l'enfant, pour estimer que cet enseignement est irrecevable par l'enfant. Rousseau, on s'en doute, ne cherche aucunement à adapter cet enseignement au public enfantin. Pour lui, l'idée de Dieu n'est pas une idée sui generis : dans l'hypothèse d'une éducation selon la nature où il serait coupé de toute influence sociale (et donc aussi religieuse), il semble qu'Émile ne développerait de lui-même aucune idée de Dieu. Celle-ci lui vient donc d'une influence extérieure : c'est uniqument lorsqu'il devient nécessaire, aux yeux de Rousseau lui-même, de lui donner une éducation religieuse, au moment de l'entrée dans la vie adulte, que l'idée de Dieu lui sera proposée, non par le précepteur mais par le Vicaire de la Profession de foi. Il est remarquable que Rousseau, là encore, anticipe quelque chose de la psychologie moderne, laquelle semble tenir le sentiment religieux chez l'enfant pour un effet de la vie sociale. On lit ainsi dans un texte de jeunesse de Piaget ces propos, qui ne sont pas sans rappeler ceux de Rousseau par la fiction ici présentée :

La thèse essentielle des modernes psychologues de la religion et de la morale est, en effet, que ces réalités [religieuses] ne sont pas innées ou congénitales, mais supposent un rapport acquis entre individus. Si, par impossible, on arrivait à élever un enfant dans une île déserte, sans aucun contact avec ses parents ou avec ses semblables, il apparaît comme infiniment probable qu'il ne connaîtrait ni sentiment religieux ni conscience de l'obligation morale ${ }^{52}$.

\footnotetext{
${ }^{50}$ Voir Scardigno - Mininni, 2013, p. 204.

${ }^{51}$ Voir Brandt, 2013b, p. 22. En revanche la prolongation de ce développement après l'adolescence, et donc dans la vie adulte, fait question selon cet auteur. Signalons qu'Oser rapproche non sans à propos la question de la spécificité de la foi de l'enfant des propos de l'apôtre Paul (1 Co 13,11): "Lorsque j'étais encore enfant, je parlais comme un enfant, je pensais et raisonnais comme un enfant. Devenu homme, $\mathrm{j}$ 'ai mis fin à ce qui était propre à l'enfant » (cité par Oser, 2013 [1988], p. 20-21).

${ }_{52}$ Piaget, 1928, p. 18. Dans la suite de son propos, le jeune Piaget avance toutefois une explication du sentiment religieux d'ordre psychanalytique, selon laquelle ce dernier provient d'un "sentiment filial, dans la 'piété' que l'enfant éprouve pour ses parents » (ibid.). Cette explication n'apparaîtra plus du tout dans ses essais ultérieurs, qui seront totalement extérieurs à la question du développement religieux.
} 
La croyance n'est innée ni pour Rousseau, ni pour les psychologues modernes. Le voudraient-ils, ces derniers ne pourraient pas observer son apparition en dehors de toute transmission sociale et familiale ${ }^{53}$. Si les pages dans lesquelles Rousseau évoque directement la question de la croyance présentée aux enfants adoptent un ton si polémique, c'est précisément parce qu'aux yeux de leur auteur l'observation exacte des enfants - il a assez dit à quel point il est fier d'avoir finement observé ses petits sujets ${ }^{54}$ - confortent son analyse du problème de la croyance de l'enfant. Nous voilà ici devant la longue page dont était extrait l'exergue, page qu'il paraît préférable de ne pas démembrer :

Si j'avais à peindre la stupidité fâcheuse, je peindrais un pédant enseignant le catéchisme à des enfants; si je voulais rendre un enfant fou, je l'obligerais d'expliquer ce qu'il dit en disant son catéchisme. On m'objectera que la plupart des dogmes du christianisme étant des mystères, attendre que l'esprit humain soit capable de les concevoir, ce n'est pas attendre que l'enfant soit homme, c'est attendre que l'homme ne soit plus. À cela je réponds premièrement qu'il y a des mystères qu'il est non seulement impossible à l'homme de concevoir, mais de croire, et que je ne vois pas ce qu'on gagne à les enseigner aux enfants, si ce n'est de leur apprendre à mentir de bonne heure. Je dis de plus que, pour admettre les mystères, il faut comprendre au moins qu'ils sont incompréhensibles; et les enfants ne sont pas même capables de cette conception-là. Pour l'âge où tout est mystère, il n'y a pas de mystères proprement dits.

Il faut croire en Dieu pour être sauvé ${ }^{55}$. Ce dogme mal entendu est le principe de la sanguinaire intolérance, et la cause de toutes les vaines instructions qui portent le coup mortel à la raison humaine en l'accoutumant à se payer de mots. Sans doute, il n'y a pas un moment à perdre pour mériter le salut éternel ; mais si pour l'obtenir, il suffit de répéter de certaines paroles, je ne vois pas ce qui nous empêche de peupler le ciel de sansonnets et de pies, tout aussi bien que d'enfants.

L'obligation de croire en suppose la possibilité. Le philosophe qui ne croit pas a tort, parce qu'il use mal de la raison qu'il a cultivée, et qu'il est en état d'entendre les vérités qu'il rejette. Mais l'enfant qui professe la religion chrétienne, que croit-il ? Ce qu'il conçoit; et il conçoit si peu ce qu'on lui fait dire, que si vous lui dites le contraire, il l'adoptera tout aussi volontiers. La foi des enfants et de beaucoup d'hommes est une affaire de géographie. Seront-ils récompensés d'être nés à Rome plutôt qu'à la Mecque ? [...] Quand un enfant dit qu'il

\footnotetext{
${ }^{53}$ C'est au contraire en s'engageant dans l'observation d'enfants héritant de traditions religieuses culturelles et familiales que le psychiatre Robert Coles a donné une étude très empathique intitulée Les enfants et Dieu (voir Coles, 1993).

${ }_{54}$ Il le fit, sans doute, à l'occasion de ses emplois de pédagogue, tâche pour laquelle il convient lui-même avec franchise qu'il n'était guère fait, avouant même n'avoir usé que de trois moyens les plus inutiles avec les enfants : " le sentiment, le raisonnement, la colère »! (Rousseau, 1959 [1769], p. 267.) possible.

${ }_{55}^{5}$ Les italiques sont de Rousseau. Ils signalent qu'il tient cette citation pour une objection
} 
croit en Dieu, ce n'est pas en Dieu qu'il croit, c'est à Pierre ou à Jacques qui lui disent qu'il y a quelque chose qu'on appelle Dieu; et il le croit à la manière d'Euripide.

'Ô Jupiter! Car de toi rien sinon

Je ne connais seulement que le nom'

Nous tenons que nul enfant mort avant l'âge de raison ne sera privé du bonheur éternel; les catholiques croient la même chose de tous les enfants qui ont reçu le baptême, quoiqu'ils n'aient jamais entendu parler de Dieu. Il y a donc des cas où l'on peut être sauvé sans croire en Dieu, et ces cas ont lieu, soit dans l'enfance, soit dans la démence, quand l'esprit humain est incapable des opérations nécessaires pour reconnaître la Divinité. Toute la différence que je vois ici entre vous et moi est que vous prétendez que les enfants ont à sept ans cette capacité, et que je ne la leur accorde pas même à quinze. Que j'aie tort ou raison, il ne s'agit pas ici d'un article de foi, mais d'une simple observation d'histoire naturelle ${ }^{56}$.

On le voit, Rousseau est un polémiste redoutable, au point que la vigueur de ses attaques peut masquer la logique de son propos. Tâchons néanmoins d'esquisser celle-ci. Si l'enseignement catéchétique traditionnel est abattu d'un trait, sans le moindre effort d'ailleurs pour le comprendre ni pour y distinguer les fruits du renouvellement qu'a connu ce genre dans la tradition protestante, c'est qu'il s'agit surtout pour Rousseau de s'attaquer directement à la notion de mystère. Comprise comme étant au-dessus de la raison, celle-ci est présentée à des enfants alors qu'ils sont incapables de faire le départ entre ce qui relève de la raison et ce qui l'excède. Lorsqu'une fois de plus Rousseau s'indigne du mensonge que peut susciter dans ce cas la recherche de l'assentiment de l'enfant, il avance une distinction importante entre ce qu'il est impossible de concevoir et même de croire : signe patent que, dans sa philosophie de la religion, le concevoir précède le croire. Il apparaîtra en effet que dans la Profession de foi qui Vicaire savoyard la croyance sera fondée sur l'intelligence d'une idée de Dieu, Rousseau partant toujours de l'idée abstraite, rationnelle, pour en arriver à la croyance.

Il semble ensuite que Rousseau, paraissant faire feu de tout bois dans la polémique, quitte son sujet en s'en prenant à l'expression de la foi lorsqu'elle est présentée comme une nécessité en vue du salut. S'il est sans doute assez lâche, un lien entre les deux premiers paragraphes existe toutefois. C'est encore le caractère verbal de l'enseignement qui est visé : tout en concédant l'importance du salut, Rousseau réfute l'argument de la portée salvifique de l'expression de la foi par une reductio ad absurdum lui permettant de placer les affirmations de foi des enfants au même rang que celles

\footnotetext{
${ }^{56}$ Rousseau, 1969 [1762], p. 554-556 (l'orthographe et la ponctuation sont modernisées par endroits).
} 
des oiseaux parleurs, puisque les unes comme les autres se font sans intelligence. Le propos, où perce une ironie rappelant Montaigne, redevient plus grave la phrase d'après : le philosophe athée peut être justement blâmé de ne pas croire, puisqu'il est capable de concevoir intellectuellement ce Dieu qui est l'objet des vérités de foi ${ }^{57}$. Mais cette obligation de croire provient précisément de cette possibilité, qui fait défaut à l'enfant. L'enfant ne croit pas en Dieu quand il dit croire. Considéré indépendamment de son contexte, ce déni de la croyance de l'enfant serait choquant : de quel droit et en fonction de quelle intuition Rousseau pourrait-il nier la croyance de l'enfant? Mais le jugement fait sens dans la perspective choisie par Rousseau, où la croyance en Dieu et la capacité à concevoir des idées abstraites doivent se correspondre. Si l'enfant est incapable d'une abstraction élevée, pourquoi croit-il alors ? En raison de sa crédulité, qui tient à la fois de sa raison limitée (dont il a amplement été question), et en même temps du crédit qu'il fait à l'adulte attestant devant lui sa croyance. Il n'y a, semble-t-il, aucune condescendance dans cette affirmation de Rousseau selon laquelle l'enfant ne croit pas en Dieu mais en celui qui le lui annonce. Au contraire, Rousseau semble une nouvelle fois renvoyer à la spécificité de l'enfant : il semblerait, à le lire, que c'est la confiance qui préside à l'expression de la foi enfantine. Cette confiance n'est pas un accord sur le plan des idées - sans quoi l'enfant n'opinerait pas devant la croyance inverse lorsque l'adulte la lui présente - mais un assentiment donné à celui dont il n'y a pas de raison particulière de douter. Cet accord, l'enfant le donne par principe, ce qui signifie qu'il pourrait le donner pour n'importe quelle croyance et en toutes parties du globe. La croyance des enfants suivra donc nécessairement celle du lieu où il se trouve. Elle fait partie d'un ensemble de vérités qui sont simplement reçues.

Ce disant, Rousseau nous semble bien proche du philosophe et logicien Wittgenstein qui rapprochait, dans De la certitude, la confiance et la croyance. Pour ce dernier, en effet, les sujets adultes que nous sommes héritent d'une image du monde qu'ils n'ont besoin ni de vérifier ni d'interroger avant que n'apparaissent des raisons de douter spécifiques, qui elles-mêmes, d'ailleurs, s'appuieront sur des certitudes non examinées. Or Wittgenstein rapproche précisément cette image du monde héritée, reçue et mobilisée dans la pratique de la « façon dont on peut apprendre à un enfant à croire en Dieu ou à ne pas y croire ${ }^{58} \gg$.

\footnotetext{
${ }^{57}$ Rousseau sous-entendra quelques lignes plus loin que l'aveuglement du philosophe athée est volontaire, ce qui n'autorise pas, d'ailleurs, à le pourchasser.

${ }^{58}$ Wittgenstein, 1997 [1969], p. 51 (§ 107).
} 


\section{SAVOIR POUR CROIRE : \\ LA CONSTITUTION ÉPISTÉMOLOGIQUE \\ DE LA CROYANCE CHEZ ROUSSEAU}

Mais contrairement à Wittgenstein, qui écrivit ces lignes en 1951, Rousseau n'envisage aucunement que l'on pourrait apprendre à un enfant à ne pas croire. Il entend au contraire acheminer son lecteur vers la possibilité d'une Profession de foi qui aurait elle aussi un fondement dans la raison naturelle. La suite de l'extrait reproduit plus haut confirme que, dans sa conception philosophique de la religion, la croyance suppose la connaissance, dont Rousseau redit à la fin du texte qu'elle n'est possible qu'à partir de l'adolescence. Un raisonnement, d'ailleurs fort curieux, permet à Rousseau de repousser l'argument pouvant lui être opposé, portant que ne pas donner à l'enfant d'instruction religieuse l'exposerait à la damnation. Rousseau appelle son contradicteur imaginaire à concéder que les insensés et les enfants morts en bas âge sont réputés sauvés en l'absence de toute croyance en Dieu : il voit en cela une reconnaissance indirecte du fait que l'enfant avant l'âge de raison (en fait jusqu'à son adolescence) est incapable de concevoir la divinité. Cela tend à montrer à l'inverse que, pour tous les autres sujets croyants, la connaissance est bien la condition même de la croyance ${ }^{59}$. Le lecteur peut être surpris : il s'attendrait plutôt à ce que Rousseau concède que l'accès à Dieu ne se limite pas à la raison, puisque les insensés et les enfants sont sauvés. Rousseau n'a-t-il pas manqué là une issue en dehors de son rationalisme strict, une voie qui lui ferait considérer la possibilité de connaître Dieu par un rapport moins tributaire de représentations abstraites, un rapport accessible à l'enfant, même si le développement de son esprit n'est pas achevé ?

Toujours est-il que ces pages préparent la Profession de foi $d u$ Vicaire savoyard: elles énoncent comme cette dernière une exigence relevant de ce qu'on appellerait aujourd'hui l'épistémologie de la croyance religieuse, selon laquelle la croyance doit s'appuyer sur le caractère concevable de ce qui est $\mathrm{cru}^{60}$. Toute croyance ne peut en effet être jugée légitime, on l'a vu dans l'exemple du mystère : pour que la croyance soit acceptable, il convient qu'existent en sa faveur des motifs de crédibilité - ce qui est proprement incompréhensible par la raison étant, pour Rousseau, proprement incroyable. Il est remarquable que Rousseau persiste à vouloir donner à l'enfant,

\footnotetext{
${ }^{59}$ Nous laissons ici de côté la question de savoir si Rousseau restitue correctement cette doctrine, et qui est ce «nous » dans « Nous tenons » : s'agit-il de Rousseau lui-même, ou s'inclut-il parmi ses coreligionnaires protestants ?

${ }^{60}$ Voir par exemple l'« Épistémologie de la croyance religieuse » de Roger Pouivet (Pouivet, 2002).
} 
devenu adolescent, un témoignage religieux. Conformément à son expérience personnelle ${ }^{61}$, ce témoignage sera présenté par un adulte référent, une noble figure religieuse, à la fois sincère, humble et questionnante ${ }^{62}$. Conformément encore à ses présupposés, ce témoignage prendra la forme d'un récit à la première personne, où le narrateur, parti de sa situation personnelle, conduira son élève vers des vérités intellectuelles sur lesquelles se grefferont des croyances proprement dites ${ }^{63}$. Toute la Profession du Vicaire savoyard cherchera ainsi à établir les rares (et maigres) affirmations de foi ${ }^{64}$ qui se situent pour Rousseau à l'intersection de la connaissance et de l'engagement subjectif du croyant :

- une Volonté anime l'Univers ; son existence est hautement probable, sans être absolument certaine, d'où un premier article de foi ;

- ses Lois désignent cette Volonté comme une Intelligence, dans laquelle le sentiment intérieur décèle une Providence (deuxième article de foi);

- enfin l'homme est libre et mû par une âme immatérielle (troisième article de foi).

Dans toutes ces affirmations de foi, la croyance permet de suppléer les lacunes de la raison tiraillée entre les argumentations opposées des écoles philosophiques, d'attester un "sentiment intérieur ${ }^{65}$ " à l'égard de Dieu, et d'offrir enfin à Émile un précieux appui pour sa conduite morale dans ce monde. À l'issue de la Profession de foi $d u$ Vicaire savoyard, Rousseau peut revenir alors à son Émile. Sans se soucier de trouver une articulation moins visible, il écrit :

J'ai transcrit cet écrit, non comme une règle des sentiments qu'on doit suivre en matière de religion, mais comme un exemple de la manière dont on peut raisonner avec son élève pour ne point s'écarter de la méthode que j'ai tâché d'établir ${ }^{66}$.

Par cette Profession de foi qui s'adresse à lui en sollicitant son concours - celui de sa raison aussi bien que de son cœur -, Émile est invité à dépasser son intérêt propre et à être bon. Il sait désormais qu'il a une âme et une conscience morale venues de Dieu, et qu'il est libre de choisir le bien ${ }^{67}$. La Profession de foi a permis

\footnotetext{
${ }^{61}$ Voir Frey, 2012, p. 159.

${ }^{62}$ Il est essentiel au propos de Rousseau que le Vicaire donne l'exemple de la bonté avant même de témoigner de sa foi. Ici aussi l'exemple doit venir avant la leçon : on reconnaît un arbre à ses fruits.

${ }^{63}$ Nous ne pouvons, là encore, que renvoyer à notre précédent article : Frey, 2012, p. 161-165.

${ }^{64}$ Cf. Rousseau, 1969 [1762], p. 576-587.

${ }^{65}$ Rousseau, 1969 [1762], p. 579 et 581.

${ }^{66}$ Rousseau, 1969 [1762], p. 635. p. 442 .

${ }^{67}$ Nous rejoignons ici tout à fait le propos de Jean-Marc Lamarre : cf. Lamarre, 2013,
} 
cette transmission, là où jusqu'ici toute leçon lui venait des sens puis du monde même. La croyance a trouvé sa place, finalement éminente, dans le processus éducatif de l'enfant. Elle constitue un pari, un saut, qui ne découle pas d'une pure nécessité logique (sans pourtant être déraisonnable ${ }^{68}$ ) et qui s'avère en outre moralement profitable. Cette croyance, énoncée à la première personne en une étrange méditation métaphysique ponctuée de quelques Credo, est bien celle d'un sujet particulier, ce Vicaire désireux de présenter à un jeune homme, en proie à des incertitudes dangereuses pour la suite de sa vie, une voie vers un bonheur possible ${ }^{69}$. Une telle croyance, de type rationaliste et moral, est donc pour Rousseau appelée à se transmettre à la faveur d'une médiation toute humaine dont la Profession de foi du Vicaire savoyard est le paradigme, parce qu'elle jette un pont entre ce qu'il est possible à l'adolescent de concevoir lui-même par sa raison et ce qui peut être attesté par un aîné plus avancé que lui dans les étapes de l'existence. On notera que le lien étroit entre la confiance et la croyance perdure, mais sous une forme plus adulte : alors que l'enfant croyait en Dieu parce qu'il croyait Jacques ou Paul, confondant d'une certaine manière la cause de sa croyance (croire en Dieu parce que Jacques m'a dit qu'Il existe) et sa raison (croire que Dieu existe, parce que tel argument plaide pour son existence...), l'adolescent pourra dire qu'il croit comme Jacques que Dieu existe, parce que... La cause de la croyance, c'est-à-dire son origine dans la parole d'un autre et le crédit qu'on lui fait, peut s'articuler désormais avec les raisons que le sujet a de croire, c'est-à-dire les motifs, personnellement examinés, pour lesquels la croyance est énoncée.

Au terme de ce parcours à travers les pages d'Émile relatives à la croyance de l'enfant, la réflexion de Rousseau et les limites qui sont les siennes suscitent une dernière remarque relative à la transmission auprès de l'enfant. La radicalité du choix de Rousseau appelant à ne jamais imposer nos vues à l'enfant pour laisser son jugement se former ${ }^{70}$ a le mérite de rendre visible un paradoxe situé au cœur de l'éducation : parce que l'être de l'enfant est en devenir,

${ }^{68}$ Comme l'écrit très justement Bernardi, chez Rousseau, « on ne croit pas ce que l'on sait [...] on ne professe pas ce que l'on tient pour seulement probable [...] l'implication du sujet dans sa croyance est entière, sans restriction, elle est littéralement adhésion à la foi professée : on ne peut croire à moitié » (Bernardi, 2012, p. 88).

${ }^{69} \mathrm{C}$ 'est en effet pour témoigner du bonheur qui est le sien, et qui pourrait être celui de son jeune élève, que le Vicaire lui ouvre son cœur et entame sa profession de foi : cf. Rousseau, 1969 [1762], p. 565. On a noté dans l'étude précédente que cette Profession de foi, même si elle renvoie pour finir à la sainteté de l'Évangile, se passe en réalité fort bien d'un rapport herméneutique à son égard : cf. Frey, 2012, p. 166s.

${ }^{70}$ Encore une fois : « Nous ne savons jamais nous mettre à la place des enfants, nous n'entrons jamais dans leurs idées, nous leur prêtons les nôtres » (Rousseau, 1969 [1762], p. 434). 
elle s'efforce à la fois d'appréhender l'enfant tel qu'il est, dans sa spécificité, tout en manifestant le vœu de le voir devenir ce qu'il est appelé à être - un enfant plus âgé, puis un adolescent, un adulte enfin. Toute éducation semble ainsi l'appeler à devenir plus qu'à être, dans un continuel croisement entre le présent qu'il faut laisser s'épanouir (c'est l'insigne mérite de Rousseau que d'avoir été le tout premier à l'affirmer) et le futur, vers lequel tout être de chair s'avance. Mais Rousseau, s'il a pressenti cela, ne parvient pas à valoriser la croyance que les enfants peuvent manifester. Il n'a pas de réels égards, lui pourtant si attentif aux mots de l'enfant et à la chance que représente l'enfance dans la vie humaine, pour les croyances des enfants, car il craint plus que tout qu'elles ne soient mensonge, verbiage, idolâtrie, qu'elles ne soient puériles et le demeurent plus tard - ou que les enfants, devenus adultes, les abandonnent parce qu'ils se feront une autre idée du monde et de la raison. Sa conception de la croyance veut élever celle-ci à une dignité particulière, entre le savoir objectif et la certitude purement subjective. Mais cette conception volontairement intellectualiste court le risque de perdre de vue le fait que la croyance exprime également, et peut-être essentiellement, un sentiment de confiance envers Dieu et envers ceux qui, de façon toujours individuelle, risquée, subjective, témoignent de lui.

\section{BIBLIOGRAPHIE}

Bernardi, 2012 : Bruno Bernardi, «'Il n'y a que l'espoir du juste qui ne trompe point' : le tiers régime de la vérité dans la philosophie de Rousseau », in : Habib, 2012, p. 80-93.

Bourgeois-Gironde, 2002 : Analyse et théologie. Croyances religieuses et rationalité, éd. par Sacha Bourgeois-Gironde, Bruno Gnassounou et Roger Pouivet, Paris, Vrin, 2002.

Bouveresse, 2007: Jacques Bouveresse, Peut-on ne pas croire? Sur la vérité, la croyance \& la foi, Marseille, Agone, 2007.

Brandt, 2013a : Pierre-Yves Brandt et James Meredith Day (dir.), Psychologie $d u$ développement religieux. Questions classiques et perspectives contemporaines, Genève, Labor et Fides, 2013.

Brandt, 2013b : Pierre-Yves Brandt, «Le développement religieux : changements de paradigmes », in : Brandt, 2013a, p. 15-38.

Burgelin, 1962 : Pierre Burgelin, Jean-Jacques Rousseau et la religion de Genève, Genève, Labor et Fides, 1962.

Coles, 1993 : Robert Coles, Les enfants et Dieu. L'enfance face à Dieu, à la foi et au doute, à l'expérience mystique et à l'âme, trad. de T. Carlier, Paris, Robert Laffont, 1993 [1990]. 
Delon, 2007 : Michel Delon (dir.), Dictionnaire européen des Lumières, Paris, PUF, 2007 (1 ${ }^{\text {re }}$ éd., 1997).

Drouin-Hans, 2013 : Anne-Marie Drouin-Hans, Michel Fabre, Denis Kambouchner et Alain Vergnioux (dir.), L'Émile de Rousseau : regards d'aujourd'hui, Paris, Hermann, 2013.

Frey, 2012 : Daniel Frey, « La profession de foi de Jean-Jacques Rousseau », RHPR 92, 2012, p. 153-174.

Greisch, 2002: Jean Greisch, Le Buisson ardent et les Lumières de la raison. L'invention de la philosophie de la religion. Tome I : Héritages et héritiers du $X I X^{e}$ siècle, Paris, Cerf, 2002.

Guyot, 1962 : Charly Guyot, «La pensée religieuse de Rousseau », in : Jean-Jacques Rousseau, Neuchâtel, Éditions de la Baconnière, 1962, p. 127-151.

Habib, 2012 : Claude Habib (dir.), Éduquer selon la nature. Seize études sur Émile de Rousseau, Paris, Desjonquères, 2012.

Hager, 2007 : Fritz-Peter Hager, «Éducation, instruction et pédagogie », in: Delon, 2007, p. 429-432.

Houdé, 2013 : Olivier Houdé, La psychologie de l'enfant, Paris, PUF, 2013.

James, 1931 [1902] : William James, L'expérience religieuse. Essai de psychologie descriptive, trad. de F. Abauzit, Paris, Félix Alcan, $3^{\mathrm{e}}$ éd., 1931 (éd. originale, 1902).

Lamarre, 2013 : Jean-Marc Lamarre, «Lire l'Émile à la lumière de la Profession de foi du vicaire savoyard », in: Drouin-Hans, 2013, p. 437-442.

L'Aminot, 1996a : Tanguy L'Aminot, «Éducation », in : Trousson, 1996, p. 275-278.

L'Aminot, 1996b : Tanguy L'Aminot, « Émile (Manuscrit Favre) », in : Trousson, 1996, p. 281-283.

Loeffel, 2013 : Laurence Loeffel, «Émile, précurseur de la psychologie de l'enfant?», in : Drouin-Hans, 2013, p. 85-98.

Michon - Pouivet, 2010 : Philosophie de la religion. Approches contemporaines. Textes réunis par Cyrille Michon et Roger Pouivet, présentés et traduits par J.-B. Guillon, C. Michon, R. Pouivet et Y. Schmitt, Paris, Vrin, 2010.

Morel, 2007 : « Enfance », in : Delon, 2007, p. 459-462.

Oser, 2013 [1988] : Fritz Oser, Paul Gmünder et Louis Ridez, L'homme, son développement religieux. Étude de structuralisme génétique, trad. L. Ridez, Paris, Cerf, 2013 (éd. originale, 1988).

Perrin, 2012: Jean-François Perrin, «Une passiveté sous haute surveillance: la mémoire de l'enfant selon l'Émile », in: Habib, 2012, p. 38-52.

Piaget, 1928 : Jean Piaget, "Immanence et transcendance », in: Jean Piaget et Jean de la Harpe, Deux types d'attitudes religieuses: Immanence et transcendance. Brochure publiée par l'Association chrétienne d'étudiants de Suisse romande, 1928, p. 7-40 (nous citons la version électronique disponible sur le site de la Fondation Jean Piaget: http://www.fondationjeanpiaget.ch/fjp/site/presentation/index.php? $P R E S M O D E=1 \& D O C I D=953$ (page consultée le 15/06/2015).

Piaget - Inhelder, 2003 [1966] : Jean Piaget et Bärbel Inhelder, La psychologie de l'enfant, Paris, PUF, $19^{\mathrm{e}}$ éd., 2003 ( $1^{\mathrm{re}}$ éd., 1966).

Porset, 1996 : Charles Porset, «Profession de foi du Vicaire savoyard », in : Trousson, 1996, p. 753-756. 
Pouivet, 2002: Roger Pouivet, «Épistémologie de la croyance religieuse », in : Bourgeois-Gironde, 2002, p. 11-30.

Pouivet, 2003 : Roger Pouivet, Qu'est ce que croire ? Paris, Vrin, 2003.

Pouivet, 2006 : Roger Pouivet, «Wittgenstein et les croyances religieuses », RHPR 86, 2006, p. 357-375.

Ricœur, 2000 [1969] : Paul Ricœur, «Croyance », Dictionnaire de la philosophie, Paris, Encyclopædia Universalis - Albin Michel, 2000 (1 ${ }^{\text {re }}$ éd., 1969), p. 309-328.

Rosenberg, 1996 : Aubrey Rosenberg, «Religion », in : Trousson, 1996, p. 794-797.

Rousseau, 1959 : Jean-Jacques Rousseau, Euvres complètes. I. Les Confessions - Autres textes autobiographiques. Édition publiée sous la direction de Bernard Gagnebin et Marcel Reymond, Paris, Gallimard, 1959.

Rousseau, 1959 [1769] : Les Confessions [1769], in : Rousseau, 1959, p. 1-656.

Rousseau, 1959 [1773-1774] : Rousseau Juge de Jean-Jacques. Dialogues [1773-1774], in: Rousseau, 1959, p. 657-992.

Rousseau, 1969 : Jean-Jacques Rousseau, Euvres complètes. IV. Émile. Éducation Morale - Botanique. Édition publiée sous la direction de Bernard Gagnebin et Marcel Raymond, Paris, Gallimard, 1969.

Rousseau, 1969 [1758-1760] : Émile (Première Version - Manuscrit Favre), in : Rousseau, 1969, p. 55-238 (manuscrit non publié rédigé entre 1758 et 1760).

Rousseau, 1969 [1762] : Émile, ou De l'éducation [1762], in : Rousseau, 1969, p. 239-868.

Rousseau, 1969 [1763] : « J.-J. Rousseau, citoyen de Genève, à Christophe de Beaumont, Archevêque de Paris » [1763], in : Rousseau, 1969, p. 925-1007.

Scardigno - Minnini, 2013 : Rosa Scardigno et Guiseppe Minnini, « Le sens de la religiosité dans les différentes périodes de la vie : une approche culturelle et discursive », in : Brandt, 2013a, p. 189-219.

Sermain, 2012 : J.-P. Sermain, «La place du nourrisson dans l'Émile », in: Habib, 2012, p. 17-25.

Trousson, 1996 : Raymond Trousson et Frédéric S. Eigeldinger (dir.), Dictionnaire de Jean-Jacques Rousseau, Paris, Honoré Champion, 1996.

Vargas, 2012 : Y. Vargas, « Tout est perdu », in : Habib, 2012, p. 149-159.

Wittgenstein, 1976 [1969] : Ludwig Wittgenstein, De la certitude, trad. J. Fauve, Paris, Gallimard, 1976 (éd. originale, 1969). 